\title{
Periodic Contention-Free Multiple Access For Power Line Communication Networks
}

\author{
*Yu-Ju Lin \\ Computing and Information Sciences Department \\ 9200 University Boulevard, Charleston, SC 29423 \\ *ylin@ csuniv.edu Haniph A. Latchman \\ Electrical and Computer Engineering Department \\ University of Florida, Gainesville, FL 32611 \\ Jonathan C.L. Liu and Richard Newman \\ Computer and Information Science and Engineering \\ University of Florida, Gainesville, FL 32611
}

\begin{abstract}
-
There is industrial intent to use Powerline Communication (PLC) PLC networks in the home for delivery of multimedia data, with associated challenging quality of service $(\mathrm{QoS})$ requirements. Existing protocols for PLC, for wireless networks, or even for wired networks cannot meet these challenges efficiently. This paper proposes and analyzes a new protocol designed to provide the high QoS needed for delivery of multiple multimedia streams in a PLC environment.

The proposed protocol, Periodic Contention-Free Multiple Ac$\operatorname{cess}(\mathrm{PCF} / \mathrm{MA})^{1}$, directly addresses the issues of asymmetric communication channel, hidden nodes and near-far effects. The proposed PCF/MA performance is analyzed theoretically and its performance is simulated. The results show that 85Mbps MAC throughput is possible with $100 \mathrm{Mbps}$ channel data rate, even when there are hidden nodes in the network.
\end{abstract}

Keywords - Keywords: Power Line Communication, Reservation, Multimedia Applications, TDMA, CSMA/CA, PCF/MA, Hidden Node, Near-Far Effect Modified CSMA/CA(MCSMA/CA).

\section{INTRODUCTION}

Recently, broadband communication over Power Line Communication(PLC) networks have attracted much interest in academe and industry, not only because of their convenience (connecting PLC capable devices requires no new wires), but also because almost all electrical devices have to connect to a power outlet eventually. PLC networks make the smart home possible [1].

The HomePlug Alliance has set standards for $14 \mathrm{Mbps}$ class data transmission, namely the HomePlug 1.0 standard. The performance and reliability of HomePlug 1.0 is comparable to wireless networks using the IEEE $802.11 \mathrm{~b}$ standard[2]. However, the HomePlug 1.0 protocol is not suitable for video playback because of its limited network throughput and lack of sufficiently stringent quality of service constraints.

\footnotetext{
${ }^{1}$ This article has not yet been published on any conferences or journals at the date of Dec. 10th.
}

To support multimedia streaming for homes, the HomePlug Alliance is now developing the standard for a second generation of PLC devices capable of delivering multiple HDTV streams using newly designed chipsets adhering to the HomePlug AV standard, which supports raw data rates up to 200Mbps. The goal of HomePlug $A V$ is to enable PLC devices to deliver some two hours of HDTV video without video frame drops, while simultaneously delivering one or more other data streams of various data rates and traffic types. To achieve the above mentioned goals, the efficient cooperation of high speed PHY and MAC protocols becomes important, a feature notably absent from current existing MAC protocols, and are therefore not suitable for HomePlug AV.

From our study, we found PLC channels have some unique characteristics:

1. Devices on PLC channel tend to be stationary.

2. PLC network channels tend to be stationary.

3. From network topology point of view, it remains stationary like an ordinary fixed network

PLC network nodes and channels tend to be stationary[2],i.e., channels may be affected by the near-by electronic devices for a short period of time, the adaptation algorithm and the power line channel characteristics make the channel remains stationary from time to time. Our study also shows that the PLC channel has similar noise characteristics to a wireless network though, from a network topology point of view, it remains stationary like an ordinary fixed network (e.g., Ethernet network).

The PLC channels are in some ways similar to wireless channels - both of them face hidden node problems, near-far effects and other channel imperfections. However, the channel conditions are more severe in PLC channel. To conquer noisy channel, an OFDM is used and a tone map is evaluated before starting a new conversation between two devices, these two processes 
cause asymmetric PLC channel as depicted on Fig.1. In this figure, the noise generator close to Device1 affects the useable tone map that it can use, results in an inferiors bandwidth. When the noise is loud enough, the Devicel will have difficulties to hear from Device2.

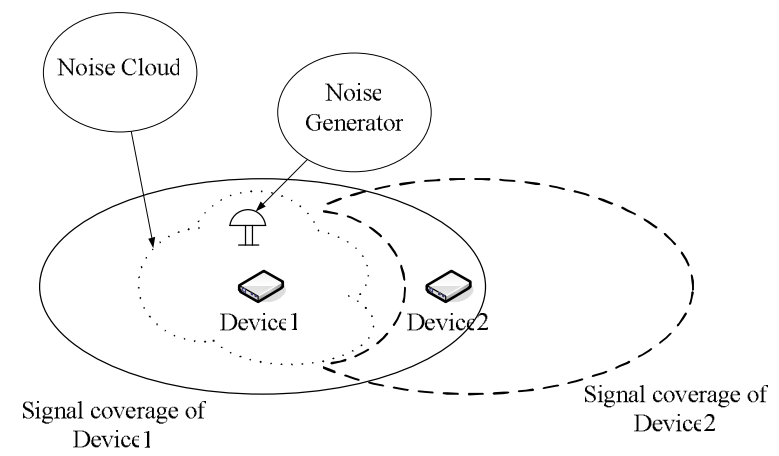

Fig. 1. Asymmetric PLC channel caused by nearby noise generator

The asymmetric PLC channel also affects usable protocols that can be used to solve hidden node problems. For example, the RTS/CTS scheme assumes that the communication channel is symmetric such that the virtual carrier sense can be received on all near-by devices. However, in asymmetric communication channel, devices that are affected by the nearby noise generator like Device1 in Fig. 1 cannot hear from Device 2 would make RTS/CTS fails, since devices that did not receive RTS may still send signals while Device 2 is transmitting.

It is unlikely that simply applying protocols designed for another medium would result in good performance in the PLC environment; the overhead may be too high or the assumptions about noise may be too optimistic for PLC networks.

In the light of PLC's unique characteristics, we propose a new protocol - Periodic Contention-Free Multiple Access(PCF/MA). PCF/MA is an explicit RALOHA-like protocol specifically designed for the PLC network - we propose an RTS/CTS-like scheme in the reservation stage to prevent hidden node problems, and a delayed NACK mechanism to conquer near-far effect. The reservation can be a permanent reservation to reduce competition in the reservation stage or MAC protocol data unit(MPDU) based reservation to provide flexibility. Performance of the proposed protocol is evaluated by event driven computer simulation and by mathematical analysis. The simulation results show that $85 \mathrm{Mbps}$ MAC throughput under 100Mbps channel data rate can be obtained, even when there are hidden nodes in the network. To provide smooth video delivery, we propose a mathematical estimation of the required delay in playback time and the amount of playback buffer with tight bandwidth reservation.

This paper is organized as follows. A brief survey of existing protocols is given in section 2 . Section 3 provides the $P C F / M A$ methodology, analysis, performance evaluation and simulation results. The discussion and conclusion are given in section 4 .

\section{Previous Work}

Before introducing PCF/MA, we would like to discuss a few recent MAC protocol developments and their applications to PLC networks. Packet contention techniques such as Carrier Sense Multiple Access with Collision Avoidance(CSMA/CA) and ALOHA find widespread use in data communications, including the first generation PLC networks. Both have the ability to serve a large number of terminals. While they function with little to no coordination, packet contention results in unpredictable behavior such as unfairness and possibly long delays, which make them unsuitable for delaysensitive packet delivery. To solve these problems, one can use packet scheduling or reservation-based methods.

Reservation-based methods were designed to remove delay-sensitive, high-rate connections from the randomaccess competition for channel time. The time domain is partitioned into reservation and data sessions; each session is then again partitioned into slots. In these methods some slots are reserved for specific stations. Other stations are restrained from using a reserved slot. Generally, these MAC schemes can be categorized according to whether the reservation is done implicitly or explicitly.

Packet Reservation Multiple Access (PRMA) is a centralized and slotted multiple access protocol that allows voice and data sources to share the same access channel at the talkspurt level, targeted for wireless local area networks [4]. PRMA utilizes speech on-off activity to improve bandwidth efficiency and system capacity. Though PRMA is an implicit reservation-based algorithm, between talkspurts stations must contend for reservations, leading to packet loss and degraded speech quality. It also relies on central control to broadcast an ACK at the end of each slot, hence it may not be appropriate for a home ad-hoc environment. A few modified PRMA protocols such as D-PRMA were proposed to support mobile ad-hoc environments. These emphasize talkspurt-level packet reservation without relying on a central entity. They also try to deal with hidden/exposed node problems by asserting an RTS/CTS-like dialog between sender and receiver.

Hidden node and near-far effects make implicit reservation difficult in PLC networks. Impaired receivers may not agree on the current network state. Further, as the PLC network is targeted to be an in-home networking infrastructure, it is not desirable to have a central control device just for medium access, especially if the central control device may become a performance bottleneck and a single failure point of a network. Lack of 
a central control device makes collision detection even more difficult since PLC devices are not able to detect signal collisions during transmission.

Explicit reservations like "Five-Phase Reservation Protocol"'5] try to implement a dynamic parallel reservation with arbitrary scalable network size. The five phases mentioned in the literature are: Reservation Request(RR); Collision Report(CR); Reservation Confirmation(RC); Reservation Acknowledgement(RA); Packing and Elimination (P/E). However, this protocol makes assumptions inapplicable to the PLC environment. First, the protocol works on a 2 hops radius network, with perfect timing. Second, near-far effects do exist in the PLC network especially when a robust signal modulation is applied. In what follows we develop a new protocol design based on an explicit reservation scheme. Robert's reservation scheme and R-TDMA are good candidates; their merit is that a few slots are dedicated for reservation purpose and the rest of them are for data transmission. However, these schemes lack distributed control, hidden-node prevention, and ignore near-far effects, which makes them inappropriate for PLC networks. Careful re-design of Robert's and the R-TDMA protocols results in the new $\mathrm{PCF} / \mathrm{MA}$ protocol as developed in the next section.

\section{Proposed PCF/MA PROTOCOL}

Though the power line channel is similar to a wireless channel, there are some unique characteristics specific to the power line channel. Firstly, the characteristics of the PLC channel in a home are in general steady over time rather than dramatically changing as in the wireless channel, i.e., while the attenuation of the signal may be affected by near-by electronics in the short term, from a long term point of view the attenuation is almost stationary [3]. This makes long-term bandwidth scheduling possible. Secondly, the devices in PLC networks are quasi-stationary, i.e., the stations in the PLC network are not constantly moving as are devices in a wireless network, thus the bandwidth fluctuations are much less erratic [2]. These two characteristics place PLC networks in a spectrum between Ethernet networks and wireless networks in that they have the properties of a fixed network topology but with much more noise and attenuation.

The asymmetric channel also makes hidden node problems more severe. The devices that cannot hear the RTS/CTS can still affect the on going transmissions. In Fig. 2 we depicted one of the common situation in PLC channel.

The situation described in Fig. 2 is quite common consider a family came back to their home after school and work, PLC capable devices like HDTV-enabled $\mathrm{TV}$ s and computers may start to function at around the
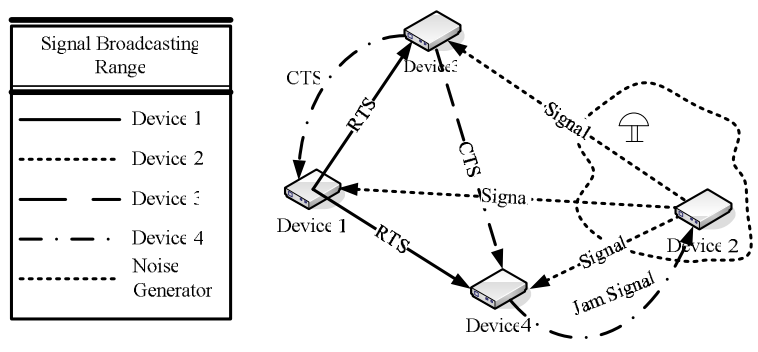

Fig. 2. RTS/CTS exchange in asymmetric PLC channel

same time, lights around the house are all turned on which generates considerably amount of noise to the PLC channel. Without proper solution to the asymmetric PLC channel, most of the devices may fail to response.

Meanwhile, delivering delay-sensitive data streams like HD-Video requires predictable MAC behavior, and contention-based protocols require more effort than contention-free/reservation-based protocols in order to provide the same functionality.

To summarize the above considerations, we propose a new protocol - PCF/MA, as stated below.

The PCF/MA protocol is a distributed, contentionfree protocol that uses a two-way handshake reservation process to establish TDMA slot assignments. The reservation process for a given node only involves nodes within a one hop radius.

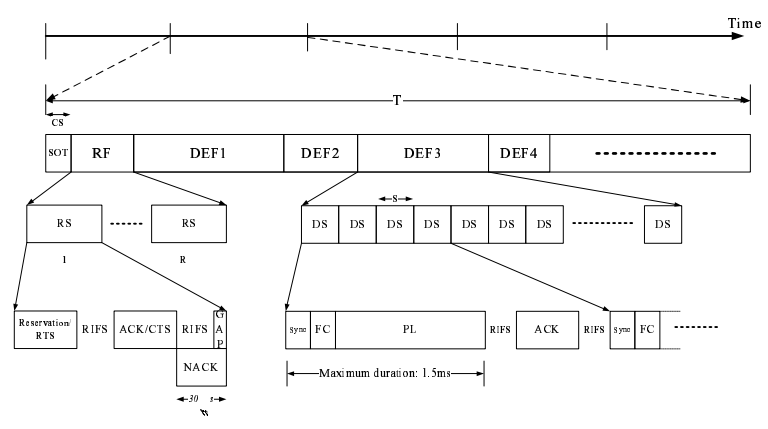

Fig. 3. PCF/MA Frame Structure

Fig. 3 shows the protocol's frame structure. Time is divided into several TDMA sessions with duration of $T \mu \mathrm{s}$. Each session is partitioned into a Reservation Frame(RF) and one or more Data Exchange Frame(DEF). Before the RF is a Start of TDMA(SOT) frame issued by all stations on the network that lasts $38.4 \mu s^{2}$ Table I summarizes the parameters used in this research.

\section{A. Reservation Process}

The RF is divided into $R$ Reservation Slots(RS). An $\mathrm{RS}$ does not correspond to a data slot as seen in most of the R-TDMA-based protocol. A RS works but rather

\footnotetext{
${ }^{2}$ The parameters we used in this paper follow those of HomePlug 1.0 as published in [7].
} 
TABLE I

HOMEPLUG 1.0 AND PCF/MA PARAMTERS

\begin{tabular}{|c|c|c|c|}
\hline & HomePlug 1.0 & PCF/MA & MCSMA/CA \\
\hline \hline SYNC & $38.4 \mu \mathrm{s}$ & $38.4 \mu \mathrm{s}$ & $38.4 \mu \mathrm{s}$ \\
\hline FC & $33.6 \mu \mathrm{s}$ & $33.6 \mu \mathrm{s}$ & $33.6 \mu \mathrm{s}$ \\
\hline RIFS & $26 \mu \mathrm{s}$ & $26 \mu \mathrm{s}$ & $26 \mu \mathrm{s}$ (SIFS) \\
\hline DEL & $72 \mu \mathrm{s}$ & $72 \mu \mathrm{s}$ & $72 \mu \mathrm{s}$ \\
\hline $\begin{array}{c}\text { Max MPDU } \\
\text { Duration }\end{array}$ & $1.5 \mathrm{~ms}$ & $1.5 \mathrm{~ms}$ & $1.5 \mathrm{~ms}$ \\
\hline CRS & $35.84 \mu \mathrm{s}$ & $200 \mu \mathrm{s}(\mathrm{RS})$ & $35.84 \mu \mathrm{s}$ \\
\hline DIFS & $35.84 \mu \mathrm{s}$ & - & $35.84 \mu \mathrm{s}$ \\
\hline $\begin{array}{c}\text { TDMA } \\
\text { Session } \\
\text { Length }\end{array}$ & - & $T \mu \mathrm{s}$ & - \\
\hline $\begin{array}{c}\text { Reservation } \\
\text { Slots }\end{array}$ & - & $R$ & - \\
\hline SOT & $38.4 \mu \mathrm{s}(\mathrm{SYNC})$ & $38.4 \mu \mathrm{s}$ & - \\
\hline
\end{tabular}

serves as a period of time for making a reservation. This process exchanges Reservation Packets(RPs) and ACKs. An RP contains the Source Address(SA), Destination Address(DA), Starting reserved Slot number(SS) and Total reserved Slots(TS) as depicted in Fig. 4.

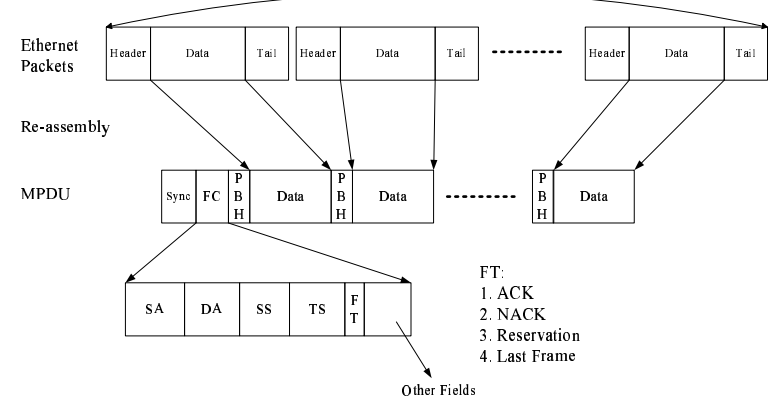

Fig. 4. MPDU Process and Format

If a node wants to reserve a DEF, it first listens to the network for at least one TDMA session. During this period, it monitors network activity and learns the reservations of each station. The node stores the knowledge into an internal table. When the RF begins, it chooses a random RS and broadcasts an RP to the networks. The destination receives and compares the request with its internal table. If the reservation does not conflict with previous reservations, the node sends an ACK to the transmitter and completes the reservation process. Other stations in the network also listen and broadcast a NACK packet to jam the possible ACK signal if they detect a conflict. If a collision/NACK happens, the requester waits for next reservation opportunity.

The data exchange period is divided into several $N$ data slots(DSs), each with a duration of $S \mu s$. A DEF is composed of several DSs. The length of a DEF is decided by the TS field in owner's RP. A DEF always begins at the start of a DS.

If a node successfully reserves a period of time for transmission, it listens and waits until its DEF time, then starts to transmit MAC Protocol Data Units(MPDUs).
If the receiver receives a successful MPDU, it will send an ACK. The sender can continue until the end of its reserved DEF.

At the end of a transmission, the sender broadcasts a "Last Frame" MPDU to the network. The receiver also broadcasts a "Last Frame" MPDU to eliminate possible hidden node problems and to allow all nodes to update their tables. When a station ungracefully terminates its connection, affected stations (usually the receiver) also broadcast a "Last Frame" MPDU during the reserved slots to synchronize tables with other stations.

MPDUs combine several Ethernet packets belonging to the same path into a jumbo packet to increase overall efficiency. The original Ethernet packet header and trailer is removed and a new small header called "PHY Block Header"( $\mathrm{PBH})$ containing the sequence number of the original packet is added to indicate the order of the packet. After this process, the original Ethernet packet becomes a new block called a PHY Block(PB). The process is depicted in Fig. 4.

Several PBs are then combined into a jumbo packet with a common header to become an MPDU ready for transmission. Based on the current transmission speed, as many Ethernet packets as possible are combined until transmission duration reaches $1.5 \mathrm{~ms}$.

A robust signal modulation is required due to the attenuation and noise on PLC channels. However, this makes detecting packet collision difficult - the signal demodulation process may interpret contending packets as noise and remove the noise to restore the intended signal! This phenomenon causes near-far effects and hidden-node problems.

A common solution to the hidden-node problem is the use of an RTS/CTS handshake before data transmission. The purpose of RTS/CTS is to notify nearby stations of the incipient data transmission period so that those who are not involved in the data exchange will avoid the channel during that period of time. Signal reception of mobile devices in wireless networks usually suffers from nearby activities, reception is expected to be constantly changing, thus exchanging RTS/CTS packets before every data transmission is required.

In PLC networks, we adopt a similar RTS/CTS scheme but only in the reservation stage. In the RF, nodes want to make reservations broadcast RPs to the network. The RPs also act as "Request To Send" signals as in the RTS/CTS scheme. Nodes that receive this packet avoid the reserved period. Nodes outside the broadcasting range may not be aware of the reservation resulting in inconsistent databases. If they do not want to make new reservations, the inconsistency become irrelevant. If one of the nodes wants to make a new reservation that conflicts with the schedulted reservations, all nodes that hear this RP broadcast NACK. 
Though the probability that the receiver will receive a strong NACK, there are chances that the NACK may not be heard by the transmitter because the near-far effect, a delayed NACK scheme is applied as shown in Fig 3.

\section{Approximate Performance AnAlysis And Simulation RESUlTS}

To calculate the maximum throughput, we assume there are always data to send for each node. We define efficiency as the ratio of time spent on transmitting payload to the total time spent on the whole data exchange process. The parameters we used in the calculation is summarized in Table I.

The minimum reservation slot time can be obtained by adding a RP duration, two RIFS, an ACK duration and a $4 \mu s$ gap which leads to $(72+26+72+26+4) \mu s=$ $200 \mu \mathrm{s}$. A successful packet transmission requires an MPDU, an ACK and two RIFSs. The total time required for this process is $1624 \mu \mathrm{s}$ when sender sends a maximum length MPDU. The total DAE slots in a TDMA session can be calculated by $\left\lfloor\frac{T-200 R-29}{s}\right\rfloor$ assuming $R$ reservation slots. If there are $m$ transmitters, and the bandwidth is evenly distributed to all transmitters, then a transmitter can have $b$ slots, where

$$
b=\frac{\left\lfloor\frac{T-200 R-29}{s}\right\rfloor}{m} \text { slots }
$$

The total allowed transmitting time for each data stream can be calculated by $b \times s$. A sender can transmit $p$ maximum sized MPDUs in a TDMA session, where

$$
p=\frac{\left\lfloor\frac{T-200 R-29}{s}\right\rfloor s}{1624 m}
$$

Since the maximum MPDU has duration of $1.5 \mathrm{~ms}$ and the overhead of a MPDU is $72 \mu \mathrm{s}$, thus the total time spent on data transmission for each node can be calculated by

$$
\left\lfloor\frac{\left\lfloor\frac{T-200 R-29}{s}\right\rfloor s}{1624 m}\right\rfloor 1428 \mu \mathrm{s}
$$

Since there are $m$ nodes, the protocol efficiency $E$ can be calculated by

$$
E=\frac{\left\lfloor\frac{\left\lfloor\frac{T-200 R-29}{s}\right\rfloor s}{1624 m}\right\rfloor 1428 m}{T}
$$

If we ignore the floor $(\lfloor\cdot\rfloor)$ operation in Eq. 1, we can calculate the maximum efficiency $E_{\max }$ as follows:

$$
\begin{aligned}
& E_{\text {max }}= \frac{\left(\frac{\left(\frac{T-200 R-29}{s}\right) s}{1624 m}\right) 1428 m}{T} \\
&= \frac{(T-200 R-29) 1428}{1624 T} \\
&= \frac{0.88(T-200 R-29)}{T} \\
& \text { When } T \rightarrow \infty \\
& E_{\max }=88 \%
\end{aligned}
$$

One must note that each DEF starts from the beginning of a DS; if a node does not fully utilize the reserved DS, the efficiency will decrease.

From Eq. 1 we learned that the efficiency of PCF/MA protocol is determined by $R, m$ and $T$, where $m$ is dynamic. To obtain the optimal performance, we derived a few mathematical forms as follows.

To minimize fixed overhead, a small $R$ would be desirable considering the number of contenders is relatively small since all reservations are persistent. However, a small $R$ could make the system unstable when the number of contenders increases. For example, let $R=1$ and there are two contenders at one instance, none of the contenders will be able to make reservation. NOTE - this depends on how backoff is handled.

It is obvious that when the number of new data streams $n$ is larger than the available reservation slots $r$, at least two stations will have a reservation packet collision. We call this over-saturation. However, since the reservation session comes every $T \mu s$, as long as the number of new data streams is less than 1 every TDMA session and at least 1 data stream successfully makes a reservation, the waiting queue should converge. When there are $r$ new data streams, this problem can be described as the probability that at least one station does not conflict with other stations. To determine this probability, we derive the following calculations.

The permutations $P$ of choosing $r$ numbers from $n$ numbers is

$$
P=r^{n}
$$

The generation function $G(x)$ corresponding to the random choice of $n$ numbers from $r$ numbers with unlimited repetition can be written as

$$
\begin{aligned}
& G(x)=\left(1+x+\frac{x^{2}}{2 !}+\frac{x^{3}}{3 !}+\cdots\right)^{r} \\
& =\quad e^{r x} \\
& =\sum_{n=0}^{\infty} \frac{r^{n}}{n !} x^{n} \text {, where } n \geq r
\end{aligned}
$$

We want to know the probability that at least one RP does not conflict with others. That is, at least one node chooses a random number that is different than the others. We can first calculate the permutations $p$ of choosing $n$ numbers from $r$ numbers such that a number is chosen 0 times, 2 times, 3 times...., then calculate $1-p / P$ to get the desired probability. The generation 
function of $p$ with unlimited repetition can be written as

$$
\begin{aligned}
& g(x)=\quad\left(1+\frac{x^{2}}{2 !}+\frac{x^{3}}{3 !}+\cdots\right)^{r} \\
& =\quad\left(e^{x}-x\right)^{r} \\
& =\quad \sum_{i=0}^{r}\left(\begin{array}{c}
r \\
i
\end{array}\right)(-1)^{i} x^{i} e^{(r-i) x} \\
& =\sum_{i=0}^{r}\left(\begin{array}{c}
r \\
i
\end{array}\right)(-1)^{i} x^{i} \sum_{j=0}^{\infty} \frac{1}{j !}(r-i)^{j} x^{j} \\
& =\sum_{j=0}^{\infty} \frac{1}{j !} \sum_{i=0}^{r}(-1)^{i}\left(\begin{array}{c}
r \\
i
\end{array}\right)(r-i)^{j} x^{(i+j)} \\
& =\quad \sum_{j=0}^{\infty} \frac{1}{j !}\left((-1)^{0}\left(\begin{array}{c}
r \\
0
\end{array}\right)(r-0)^{j} x^{j}\right)+ \\
& (-1)^{1}\left(\begin{array}{l}
r \\
1
\end{array}\right)(r-1)^{j} x^{(j+1)}+ \\
& (-1)^{2}\left(\begin{array}{c}
r \\
2
\end{array}\right)(r-2)^{j} x^{(j+2)}+ \\
& (-1)^{3}\left(\begin{array}{l}
r \\
3
\end{array}\right)(r-3)^{j} x^{(j+3)}+ \\
& \left.\cdots+(-1)^{r}\left(\begin{array}{r}
r \\
r
\end{array}\right)(r-r)^{j} x^{(j+r)}\right) \\
& =\quad \frac{1}{0 !}\left[(-1)^{0}\left(\begin{array}{c}
r \\
0
\end{array}\right)(r-0)^{0} x^{0}+\right. \\
& (-1)^{1}\left(\begin{array}{l}
r \\
1
\end{array}\right)(r-1)^{0} x^{(0+1)}+ \\
& (-1)^{2}\left(\begin{array}{c}
r \\
2
\end{array}\right)(r-2)^{0} x^{(0+2)}+\cdots \\
& \left.(-1)^{r}\left(\begin{array}{c}
r \\
r
\end{array}\right)(r-r)^{0} x^{(0+r)}\right]+ \\
& \frac{1}{1 !}\left[(-1)^{0}\left(\begin{array}{c}
r \\
0
\end{array}\right)(r-0)^{1} x^{1}+\right. \\
& (-1)^{1}\left(\begin{array}{c}
r \\
1
\end{array}\right)(r-1)^{1} x^{(1+1)}+ \\
& (-1)^{2}\left(\begin{array}{c}
r \\
2
\end{array}\right)(r-2)^{1} x^{(1+2)}+\cdots \\
& \left.(-1)^{r}\left(\begin{array}{r}
r \\
r
\end{array}\right)(r-r)^{1} x^{(1+r)}\right]+ \\
& \frac{1}{n !}\left[(-1)^{0}\left(\begin{array}{c}
r \\
0
\end{array}\right)(n-2)^{n} x^{n}+\right. \\
& (-1)^{1}\left(\begin{array}{l}
r \\
1
\end{array}\right)(r-1)^{n} x^{(n+1)}+ \\
& (-1)^{2}\left(\begin{array}{c}
r \\
2
\end{array}\right)(r-2)^{n} x^{(n+2)}+\cdots \\
& \left.(-1)^{r}\left(\begin{array}{c}
r \\
r
\end{array}\right)(r-r)^{n} x^{(r+n)}+\cdots\right]
\end{aligned}
$$

Since we are looking for the coefficient of $x^{n} / n$ !, rearranginge Eq. 4 yields

$$
\begin{aligned}
& g(x)=\quad \frac{1}{0 !}(-1)^{0}\left(\begin{array}{c}
r \\
0
\end{array}\right)(r-0)^{0} x^{0}+ \\
& {\left[\frac{1}{0 !}(-1)^{1}\left(\begin{array}{c}
r \\
1
\end{array}\right)(r-1)^{0}+\right.} \\
& \left.\frac{1}{1 !}(-1)^{0}\left(\begin{array}{c}
r \\
0
\end{array}\right)(r-0)^{1}\right] x^{1}+ \\
& {\left[\frac{1}{0 !}(-1)^{2}\left(\begin{array}{r}
r \\
2
\end{array}\right)(r-2)^{0}+\right.} \\
& \frac{1}{1 !}(-1)^{1}\left(\begin{array}{l}
r \\
1
\end{array}\right)(r-1)^{1}+ \\
& \left.\frac{1}{2 !}(-1)^{0}\left(\begin{array}{r}
r \\
0
\end{array}\right)(r-0)^{2}\right] x^{2}+ \\
& {\left[\frac{1}{0 !}(-1)^{n}\left(\begin{array}{c}
r \\
n
\end{array}\right)(r-n)^{0}+\right.} \\
& \frac{1}{1 !}(-1)^{(n-1)}\left(\begin{array}{l}
r \\
n-1
\end{array}\right)(r-(n-1))^{1}+ \\
& \cdots+ \\
& \left.\frac{1}{r !}(-1)^{(n-r)}\left({ }_{r-b}^{r}\right)(n-(n-r))^{r}\right] x^{b}+
\end{aligned}
$$

The coefficients of $x^{n} / n$ ! become

$$
\begin{array}{cc}
p= & r !\left[\frac{1}{0 !}(-1)^{n}\left(\begin{array}{c}
r \\
n
\end{array}\right)(r-n)^{0}+\right. \\
& \frac{1}{1 !}(-1)^{(n-1)}\left(\begin{array}{c}
r \\
n-1
\end{array}\right)(r-(n-1))^{1}+\cdots+ \\
& \left.\frac{1}{r !}(-1)^{(n-r)}\left(\begin{array}{c}
r \\
r-n
\end{array}\right)(r-(n-r))^{r}\right] \\
= & r ! \sum_{l=0}^{r} \frac{1}{l !}(-1)^{(n-l)}\left(\begin{array}{l}
r \\
n-l
\end{array}\right)(r-(n-l))^{l}
\end{array}
$$

From Eq. 6 and 3, we obtain the desired probability as

$$
1-\frac{p}{P}=1-\frac{n ! \sum_{l=0}^{r} \frac{1}{l !}(-1)^{(n-l)}\left(\begin{array}{c}
r \\
n-l
\end{array}\right)(r-(n-l))^{l}}{r^{n}}
$$

To further simplify Eq. 4, we do the following assumptions

$$
\begin{gathered}
n=i+j \\
S(j, r)=\frac{1}{r !} \sum_{i=0}^{r}(-1)^{i}\left(\begin{array}{c}
r \\
i
\end{array}\right)(r-i)^{j}
\end{gathered}
$$

Where $S(j, n)$ is the Stirling number of the second kind. We can then reduce Eq. 4 to

$$
g(x)=\sum_{j=0}^{\infty} \frac{r !}{j !} S(j, r) x^{n}=\sum_{j=0}^{\infty} \frac{n ! r !}{j !} S(j, r) \frac{x^{n}}{n !}(9)
$$

The coefficient of $x^{n} / n$ ! is the desired permutation p. From Eq. 3 and Eq. 9, the probability of at least one reservation packet does not conflict with others can then be represented as

$$
1-\frac{p}{P}=1-\frac{\sum_{j=0}^{\infty} \frac{n ! ! ! !}{j !} S(j, r)}{r^{n}}
$$

The term $\sum_{j=0}^{\infty} \frac{n ! r !}{j !} S(j, r)$ in Eq. 10 is a subset of $r^{n}$, it is obvious that Eq. 10 will converge when $r \rightarrow \infty$. Our theoretical and simulation results will present at the later part of this paper.

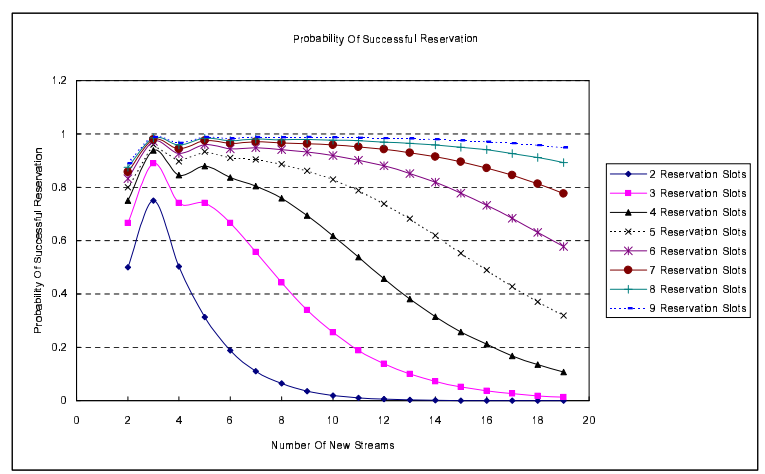

Fig. 5. Probability Of Successful Reservation

To verify formula 7 , we simulated a constant number of contenders. The simulator simulates one million 
reservation sessions, the number of contenders are the same from session to session. The simulator counts the event that at least one contender successfully makes its reservation, which is needed to avoid instability. The simulation results are shown in Fig. 5

From the simulation results, we chose $r=8$ as a conservative parameter for PCF/MA.

We also modified the CSMA/CA protocol used in the 802.11b to a version suitable for PLC. The Modified CSMA/CA (MCSMA/CA) uses the same MPDU procedure as in PCF/MA and the duration is also limited to $1.5 \mathrm{~ms}$. We follow the parameters of HomePlug 1.0 as listed in the Table I.

To investigate the performance impact with and without the near-far effect, we choose a near-far effect model described as the probability a receiver captures the desired packet $\left(P_{c a p}\right)$ in the presence of interfering $\operatorname{packets}\left(R_{i}\right)$

$$
\begin{array}{r}
P_{\text {cap }}=\text { Probability }\left(\frac{R_{d}-\sum_{i=0}^{m} R_{i}}{R_{d}}\right) \\
\text { when } \frac{\sum_{i=0}^{m} R_{i}}{R_{d}} \geq C_{T H}
\end{array}
$$

where $R_{d}$ is the received power level of the desired packet and $C_{T H}$ is the capture threshold.

Since the the packet loss rate is high $\left(10^{-3}\right.$ or higher), silent re-transmission is required when the receiver receives corrupt packets. This requires data streams to allocate extra bandwidth above the estimated bandwidth.

Assume packet loss rate is $P$ and the number of transmission attempts is $R$. Suppose at one instance, the sender sends $N$ PBs in a MPDU, the amount of over allocation for next transmission is $N P$. Thus for quasierror free environment with unlimited retries, we need to overallocate $(O)$ resources by

$$
\begin{array}{rlc}
O & = & \left\lceil N P+N P^{2}+\cdots+N P^{R}+\cdots\right\rceil \\
& = & \left\lceil N P\left(1+P+P^{2}+\cdots+P^{R-1}+\cdots\right)\right\rceil \\
& = & \left\lceil N P\left(\frac{1}{1-P}\right)\right\rceil
\end{array}
$$

When $P \ll 1$, Eq. 13 becomes $\lceil N P\rceil$.

Simulation results based on $10^{6}$ TDMA sessions closely match the mathematical calculation.

Event-driven simulation with Always On data streams was used to investigate PCF/MA performance and to compare PCF/MA with MCSMA/CA.

Fig. 6 shows PCF/MA has maximum performance gain over MCSMA/CA of about $100 \%$ when the number of contenders is large $(m \geq 60, T=100)$. It also shows that PCF/MA with large $T$ not only increases throughput, but also increases the number of possible contenders $(m)$. The reason that $T$ decides $m$ can be inferred from Eq. 2, which shows that for a given $T$, the floor operations cause the useful time of each MPDUs to become less as $m$ increases.

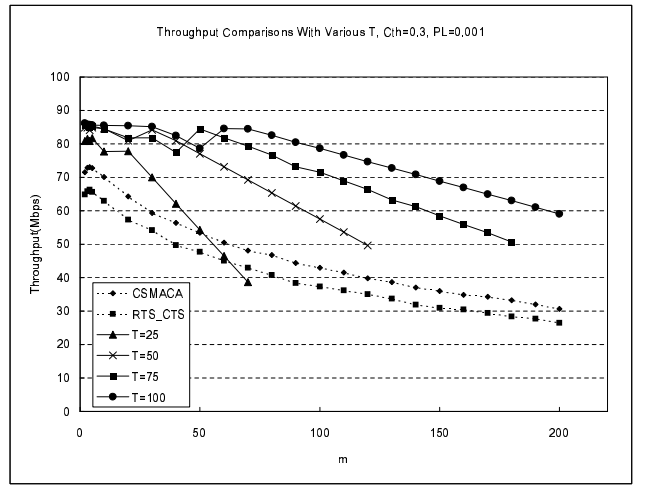

Fig. 6. PCF/MA at Various $T$ Versus Modified CSMA/CA Throughput Comparisons

To discover the performance of PCF/MA in delivering DVD video streams, we assume a single PLC station acts as a video server for several destinations. We assume the channel data rate remains stable throughout the whole video display length. We assume the video source is able to supply one video frame immediately after the request is received and the server will start to transmit MPDUs once it gets permission to transmit. The client moves the received MDPUs to the playback buffer and re-assembles them to a normal video frame before playback. If a video frame is still in transit when it is time to display, the video frame is counted as a missed deadline video frame. The client will try to display the video frame $33.3 \mathrm{~ms}$ later. The miss deadline count keeps incrementing until the video frame is successfully displayed.

To smooth video streams, a certain amount of playback buffer $(B)$ is required. When the buffer level is lower than the predefined buffer threshold $\left(B_{T H}\right)$, the client sends requests to the server to ask for more video frames. If at time $T$, we have $B_{T}>B_{T H}$, the server stops transmitting MPDUs.

To make sure the missing video frames are not caused by the protocol in use but by another factor, we investigated the relationships between the playback buffer size at a specific time $T\left(B_{T}\right)$, playback delay $(D)$, average frame $\operatorname{size}\left(V_{a}\right)$, maximum video frame $\operatorname{size}\left(V_{\max }\right)$ channel bandwidth $\left(B_{c}\right)$ and the effective bandwidth $\left(B_{w}\right)$ reserved for this video stream.

At time $T$, the buffer level at the client side can be calculated by

$$
B_{T}=B_{w} D+B_{w} T-\sum_{i=1}^{30 T} V_{i}
$$

where $V_{i}$ is the data size of frame $i$. If we assume $T$ is relatively large, such that $V_{i}=V_{a}$ and Eq. 14 becomes

$$
B_{T}=B_{w} D+B_{w} T-30 T V_{a}
$$

At time $T$, the server encounters a Group Of Pictures 
(GOP) with large video frames of size $V_{\max }$ bytes. Assume the number of video frames in a GOP is 15 , then it takes $t=\frac{15 V_{\max }}{B_{w}}$ second to deliver this GOP. During this $t$ time frame, the client consumes $N=30 \times \frac{15 V_{\max }}{B_{w}}$ video frames, i.e., the client consumes $N \times V_{\max }^{B_{w}}=$ $\frac{450 V_{\max }^{2}}{B_{w}}$ bytes. Meanwhile it accumulates $15 \times V_{\max }$ bytes. Suppose there are $G$ such GOPs, the Eq. 15 becomes

$$
\begin{gathered}
B_{T}=B_{w} D+B_{w} T-30 T V_{a}+ \\
G\left(15 V_{\max }-\frac{450 V_{\max }^{2}}{B_{w}}\right) \geq 0
\end{gathered}
$$

In order to prevent video frames from missing their deadlines, we must make Eq. 16 always larger than zero. To help us analyze Eq. 16, we re-arranged it as follows:

$$
\begin{gathered}
B_{T}=\left(B_{w} D-G\left(\frac{450 V_{\max }^{2}}{B_{w}}-15 V_{\max }\right)\right)+ \\
\left(B_{w}-30 V_{a}\right) T \geq 0
\end{gathered}
$$

The term $\left(B_{w} D-G\left(\frac{450 V_{\max }^{2}}{B_{w}}-15 V_{\max }\right)\right)$ in Eq. 17 is the accumulated buffer size during the playback delay time plus buffer loss during transmitting large video frame GOPs. The term $\left(B_{w}-30 V_{a}\right)$ is the difference of the reserved bandwidth and the average DVD title bit rate. In real world applications, $B_{w}, V_{\max }$ and $V_{a}$ are DVD statistics thus can be easily defined.

To make $B_{T}$ always larger than zero, we conclude the following sufficient, but not required, condition:

$$
B_{w} D-G\left(\frac{450 V_{\max }^{2}}{B_{w}}-15 V_{\max }\right) \geq 0
$$

and

$$
B_{w}-30 V_{a} \geq 0
$$

If we assume the reserved bandwidth is the video average bit rate, the $D$ can be calculated as:

$$
\begin{aligned}
D & \geq \frac{G\left(450 V_{\max }^{2}-15 B_{w} V_{\max }\right)}{B_{w}^{2}} \\
& =\frac{G\left(450 V_{\max }^{2}-450 V_{a} V_{\max }\right)}{\left(30 V_{a}\right)^{2}} \\
& =\frac{G\left(V_{\max }^{2}-V_{a}\right)}{2 V_{a}^{2}}
\end{aligned}
$$

Since the minimum playback buffer size should satisfy $B=B_{w} D$, from Eq. 19, we can derive the required buffer size as

$$
B \geq G\left(\frac{30 V_{\max }^{2}}{B_{w}}-V_{\max }\right)
$$

Since the playback delay $D$ and playback buffer is for the worst scenario in the DVD playback, the $G$ should be large enough to satisfy this situation; thus we set $G=60$. To verify our formula, we simulated a video server/client assuming no other data streams on the network where the client has an unlimited buffer. The efficiency of PCF/MA is about $80 \%$ when $T=25 \mathrm{~ms}$. The reserved bandwith for PCF/MA is $9.44 \mathrm{Mbps}$, the observed throughput is $7.45 \mathrm{Mbps}$ which is close to average bit rate. We slowly increase delay playback time $D$, and observe if the simulation drops video frames. If it drops video frames, we record the maximum buffer it accumulates before dropping. The total simulated time is 20 minutes which is about one sixth of the original movie length.

In simulating MCSMA/CA protocol, we follow the same procedure except the channel data rate is assigned as $15.5 \mathrm{Mbps}$ and the observed throughput is around 7.58Mbps. The simulation results are shown in Fig. 7.
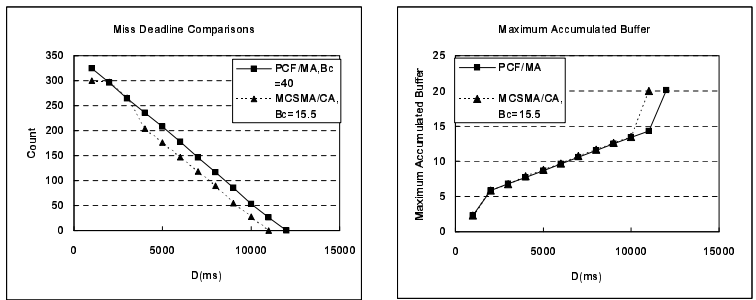

Fig. 7. Video Playback Performance Comparisons

The simulation results show that without competition, the MCSMA/CA performs slightly better than PCF/MA (though at worse efficiency). In Fig. 7, when $D=1$, the client drops about 320 video frames and the maximum buffer accumulated is about 2Mbytes. The result shows that the delay time is too short to build up a safe buffer for future video bit rate fluctuation even with unlimited buffer. The figure also shows that the video stops dropping after we increase $D$ to 12 for PCF/MA and 11 for MCSMA/CA. In both cases, the maximum accumulated buffer size is more than 20Mbytes. This result suggests that when the delay playback time and buffer is large enough, both protocols have ample time to deliver large video frames thus no video frames were dropped.

\section{CONCLUSION}

This paper proposes a new protocol - PCF/MA for high speed PLC networks. To lower overhead caused by contention between MPDUs, we choose a contentionfree method. Through simulation, we observed the network efficiency as high as $85 \%$ at $T=100$. Theoretical analysis found that with eight reservation slots, it can provide a good contention/overhead balance. We also conducted a simulation with constant contenders that verified our analysis. For comparison purposes, we modified the widely used CSMA/CA protocol into a PLC version MCSMA/CA protocol. The simulation results show that $\mathrm{PCF} / \mathrm{MA}$ protocol has the maximum performance gain of $100 \%$ over MCSMA/CA when the 
$T=100$. Our protocol also proved to be able to support more than 200 streams at the same time.

The high speed PLC is targeted for Audio/Video applications over local area networks, however, the parameters for clients to support variable bit rate data streams remain uncertain at this stage. We analyzed the behaviors of PCF/MA protocol in delivering commercial DVD titles, and derived a relationships between required buffer, reserved bandwidth, delay playback time and video statistics. We then used the estimated parameters to design a video server/client simulator in which PCF/MA and MCSMA/CA as MAC protocols were compared. The simulation results showed that PCF/MA performed much better than MCSMA/CA protocol because of its predictable behavior and low contention/overhead.

Though the PCF/MA protocol proved to be a high performance protocol, there is much work to be done. Currently, we do not implement priority classes and treat the incoming queue length of the PCF/MA protocol as unlimited. We will address these issues in future publications.

\section{REFERENCES}

[1] Yu-Ju Lin, Haniph A. Latchman, Minkyu Lee, Srinivas Katar, "A Power Line Communication Network Infrastructure for the Smart Home," IEEE Wireless Communications, no. 6, December 2002, pp. 104-111

[2] Yu-Ju Lin et.al., "A Comparative Performance Study of Wireless and Power Line Networks," IEEE Communications Magazine, April 2003, pp. 54-63.

[3] "Intellon Internal Document."

[4] D. J. Goodman, R. A. Valenzuela, K. T. Gayliard and B. Ramamurthi, "Packet Reservation Multiple Access for Local Wireless Communications," IEEE Transactions on Communications, Vol. 37, No. 8, Aug. 1989, pp. 885-890.

[5] Chenxi Zhu and M. Scott Corson, "A Five-Phase Reservation Protocol(FPRP) for Mobile Ad Hoc Networks," Seventeenth Annual Joint Conference of the IEEE Computer and Communications Societies. Proceedings. IEEE INFOCOM '98, Vol. 1, 29 March-2 April 1998 Pages:322 - 331

[6] Hadzi-Velkov, Z.; Spasenovski, B.; "Capture effect in IEEE 802.11 basic service area under influence of Rayleigh fading and near/far effect," The 13th IEEE International Symposium on Personal, Indoor and Mobile Radio Communications , 15-18 Sept. 2002 Pages: 172 - 176 vol.1

[7] M. K. Lee, R. E. Newman, H. A. Latchman, S. Kartar and L. Yonge, "HomePlug 1.0 powerline communication LANs - protocol description and performance results" International Journal Of Communication Systems 2003, vol. 16, pp.447-473 